\title{
Biometrics Recognition based on Image Local Features Ordinal Encoding
}

\author{
Simina Emerich \\ Department of Communications, \\ Technical University of Cluj-Napoca, \\ 28 Memorandumului, Cluj-Napoca, 400114, Romania
}

\author{
Bogdan Belean \\ National Institute for Research and Development of Isotopic \\ and Molecular Technologies, CETATEA Research Centre, \\ 67-103 Str. Donat, Cluj-Napoca, 400293, Romania
}

\begin{abstract}
In the present informational era, with the continue extension of embedded computing systems, the demand of faster and robust image descriptors is an important issue. However, image representation and recognition is an open problem. The aim of the paper is to embrace ordinal measurements for image analysis and to apply the concept for a real problem, such as biometric identification. Biometrics provides a robust solution for the identity management process and is increasingly more present in our life. To explore the textural discriminative information of images, the paper proposes a new feature extraction technique, namely, Image Local Features Ordinal Encoding.
\end{abstract}

Keywords-Biometrics; image local features; ordinal measurements; iris; dorsal hand veins

\section{INTRODUCTION}

The recent advancements achieved in computer vision, together with sensors evolution, play a key role in the development of real-world applications. A privileged area of applications where ordinal analysis and encoding procedures have been found to be relevant is pattern recognition. Biometric recognition is defined as automatic person identification based on vectors derived from biological characteristics. Ordinal measurements were employed by several biometric systems especially for signal or image feature extraction purposes, mainly because of their computational properties. A brief description of the main pattern recognition techniques based on ordinal comparisons with notable influence on image processing is further presented.

In 1990, He and Wang proposed the first method for image encoding based on the intensity value of the pixels from a local neighborhood, namely Texture Unit Number (TUN) [1]. In [2] one of the most powerful texture descriptors Local Binary Patterns (LBP) were introduced. The LBP operator compares each image pixel with its 8 neighbor pixels: if the neighbor pixel is greater or equal then the central one, a binary 1 is resulted, otherwise a binary 0 is used. Finally, a 256 values histogram is used to collect the occurrences of local patterns. To reduce the dimensionality Ojala et al. [3] observed that if only the 'uniform' patterns (where the maximum number of bit-wise changes is 2) are retained, the discrimination performance remains similar. A significant advantage of this technique is that avoids the need of using time or frequency normalization. Lately many variations were proposed in literature, starting from this methodology, LBP and its variants have been successfully used for image recognition tasks including biometrics. For example, the use of several patchbased image descriptors: Local Binary Pattern, Local Phase Quantization and Differential Excitation, has been investigated in [4] for iris recognition.

Another approach, named Local Line Binary Pattern (LLBP) determines a line binary code along with horizontal respectively vertical direction and its magnitude, and characterizes the change in image intensity such as edges and corners [5]. It was applied especially in biometric recognition systems based on hand veins structure from finger [6], palm [7] or the dorsal part [8].

Wang et al. proposed in 2011 a highly discriminative method called Local Intensity Order Pattern (LIOP) that uses the advantages offered by ordinal measurements to extract the image descriptors [9]. The input image is partitioned into square patches with odd length and then each local patch is divided into sub-regions with the same intensity. Next, a Local Intensity Order Pattern of each point is computed, based on the relationships among the intensities of its $N$ neighboring points. For each vector, a mapping is done by sorting the elements of the vector in an increasing order and assigning an integer value from 1 to $N$ !, since there are $N$ ! possible permutations. This approach explores the fact that the relative order of pixel intensities remains unchanged when the intensity changes are monotonic [10] and has been successfully applied in [11] to extract the representative information from iris texture.

Other ordinal measurements based methods proposed in literature to solve various problems such as image recognition, tracking or classification are: Ordinal and Spatial information of Regional Invariants (OSRI) [12], Multisupport Region Order-based Gradient Histogram (MROGH), Multisupport Region Rotation and Intensity monotonic invariant Descriptor (MRRID), etc. [13]. A very comprehensive study of image ordinal descriptors was recently published by Fan and Wang in [14].

The rest of the article is organized as follows. In Section II we present the proposed technique, namely Image Local Features Ordinal Encoding (ILFOE). It will be integrated, in Section III, in two biometric systems, based on iris respectively dorsal hand veins. Finally, Section IV concludes the paper. 


\section{IMAGE LOCAL FEATURES ORDINAL ENCODING}

It is well-known that a pattern recognition algorithm needs to solve the following three problems: what to measure, how to measure and how to interpret the results.

The human brain capabilities of visual pattern recognition remain poorly understood. Some recent studies state that the computational models disposed by the visual cortex are based on qualitatively comparing rather than quantitative information. According to this idea, a new method applicable for image recognition and based on ordinal measurements, is further proposed. Qualitative comparisons, associated to the relative ordering of extracted characteristics, are defined as ordinal measurements.

Usually, an automatic image classification approach contains three main modules: firstly, relevant features are extracted from images, the features are then encoded into descriptors and finally, the classification is achieved by disposing the image descriptors into a machine learning algorithm. The encoding process affects the system efficiency (speed and accuracy).

Of late years, different descriptors have been designed to improve the performance of standard histogram encoding procedure. The Bag-of-Features encoding model has been extensively explored for image recognition. The most known Bag-of-Features strategies are: Voting Based Methods (Hard Voting and Soft Voting), Fisher Kernel, Sparse Coding, Local tangent Coding, Super Vector Coding, Salient Coding, etc. [15], [16].

Further we focus on the first two step of this pipeline. The framework of the proposed ordinal image encoding algorithms is presented in Fig. 1.

The local features could be extracted from image filter responses or from image patches. The selection of the optimum filter banks is application dependent. The use of image patches is considered to be faster and less complex than using image jets [17].

The designed method, namely Image Local Features Ordinal Encoding (ILFOE), consist of representing images through the differences between patch based local features.

The intensity pixels values will be explored by different local processing algorithms on a squared or circular neighborhood. The employed techniques must satisfy several requirements: should be highly informative and should capture textural variation. The number of features resulted from each patch is preferable be fixed and small.

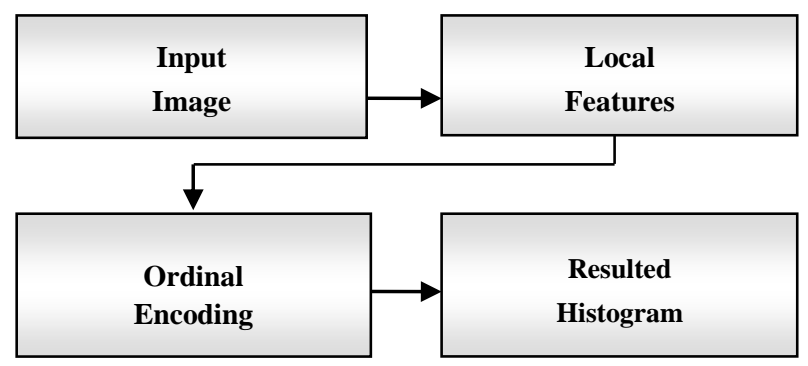

Fig. 1. Image ordinal encoding.

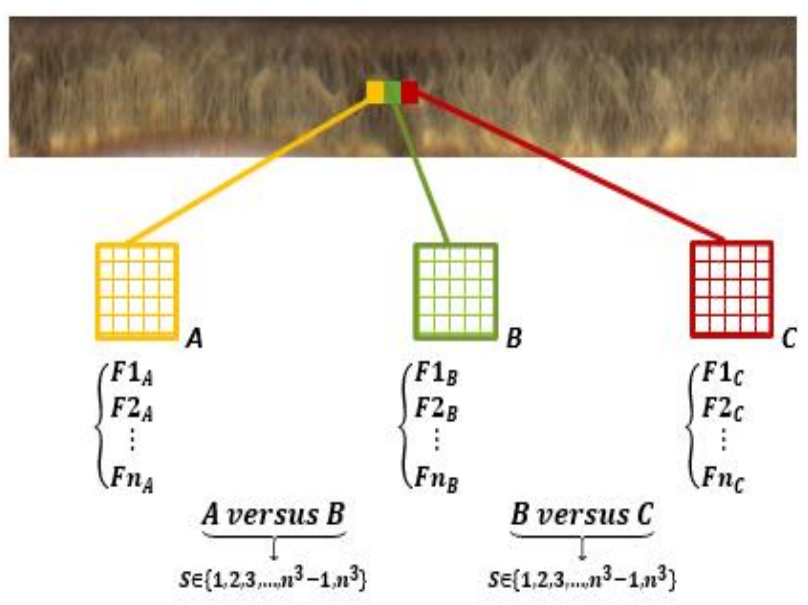

Fig. 2. ILFOE Algorithm.

The ordinal relation between pairs of features extracted from succesive patches is further investigated. The algorithm implies qualitative feature comparisions such as greater than, less than and equal. Fig. 2 summarizes the proposed ILFOE algorithm.

For each comparison, a ternary code $(+, 0,-)$ is established, as follows: if the difference between considered features is greater than a threshold value $t$, then "+" is assigned to it. A difference lesser than $-t$ is encoded with "-_" and a difference value in the range of width $t$ around zero is quantized to " 0 ".

$$
T C m=\left\{\begin{array}{cc}
+, & F m_{B}-F m_{A}>t \\
0, & -t \leq F m_{B}-F m_{A} \leq t \\
-, & F m_{B}-F m_{A}<-t
\end{array}\right.
$$

Where $F m, \overline{m=1, n}$ are local features, $A$ and $B$ are successive neighborhoods and $t$ is a predefined threshold.

The signs of the differences between adjacent neighborhoods are encoded into symbols, resulting in $n^{3}$ distinct values, where $n$ is the number of the considered local features. The proposed method converts the input image into an encoded stream of discrete numerical symbols, resulted from ordinal comparisons, as shown in Fig. 3. It is expected that the compactly extracted vectors will facilitate the matching process. The new approach is very flexible and could be adapted so that region patterns to be constructed dependent upon the image classification task.

For unidimensional signals, a similar procedure, namely TESPAR DZ has been proposed in [18] and successfully employed especially for speech analysis.

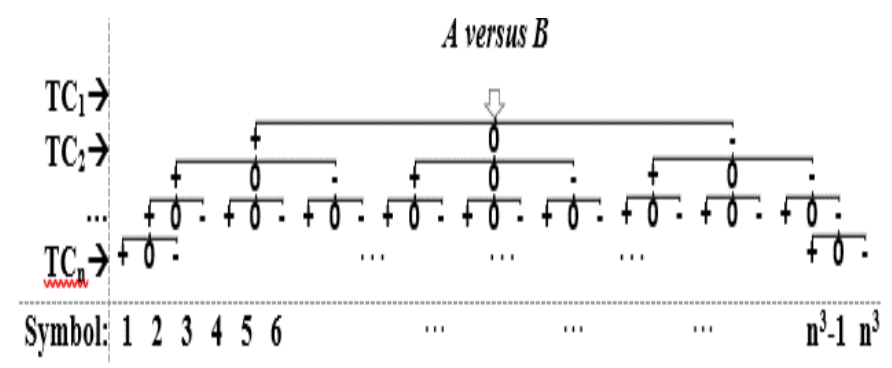

Fig. 3. Discrete numerical symbols resulted from ordinal comparisons. 


\section{DESIGNING A GENERAL FrAMEWORK FOR BIOMETRIC RECOGNITION BASED ON ORDINAL MEASURES}

The paper intent is to argue that ordinal image representation provides an appropriate solution for efficient biometric authentication. The presented method will be integrated in two recognition systems, based on iris respectively dorsal hand veins. The processing flow used to implement the biometric systems is presented in Fig. 4.

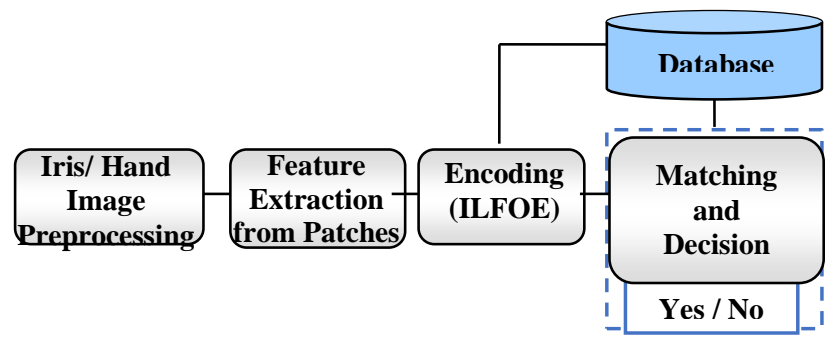

Fig. 4. Biometric system processing flow.

\section{A. Unimodal Biometrics Systems based on Iris and Dorsal Hand Veins}

In present, the identity management solutions based on both iris and hand veins recognition have received a considerable attention. Compared with others physiological traits, iris and dorsal hand veins are less susceptible to damage or forgery and remains unchanged for a long period of time.

To evaluate the iris biometric system, experiments were made on two publicly available databases: UPOL and CASIA_V1. The first one includes high resolution and texture rich color iris images, captured under visible lighting from 64 persons (3 for each eye) [19]. The second database contains greyscale images, collected from Asian persons [20]. The iris region is often covered by eyelids and eyelashes (the iris content is less than $67 \%$, for $11 \%$ of images [21]). Different experiments have been conducted by considering 93 users and 5 different images for each individual, taken from the same eye.

The inner and outer boundaries of the iris were delimited during segmentation process by the help of the circular Hough transform. The region of interest (ROI) was then unwrapped into polar coordinates and used for the feature extraction step. Since the upper and bottom of the iris, are often occluded by eyelashes or eyelids, we investigate our method on the side parts only. The half iris area ( 8 blocks) was selected between $315^{\circ}$ and $45^{\circ}$ for the right side and between $135^{\circ}$ and $225^{\circ}$ for the left side. After segmentation step, 8 blocks of same dimension are selected from the unwrapped rectangular iris image according to Fig. 5.

The biometric system based on dorsal hand veins has been designed by using the NCUT Part A database. For experimental setup, 1020 near infrared gray images collected from the left hands of 102 individuals (10 samples / user) has been considered [22]. After region of interest selection, the following techniques has been employed for image enhancement: an adaptive histogram equalization with Rayleigh distribution, followed by a median filtering and an anisotropic diffusion. The resulted image has been further divided into 9 equal blocks as shown in Fig. 6.

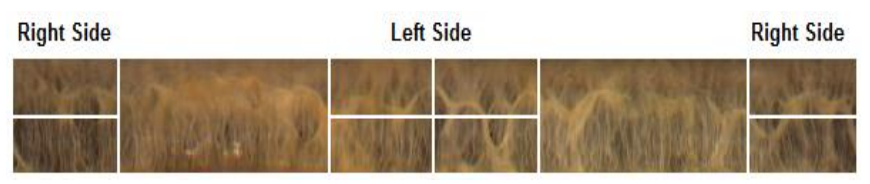

Fig. 5. Half iris area selection (8 blocks).
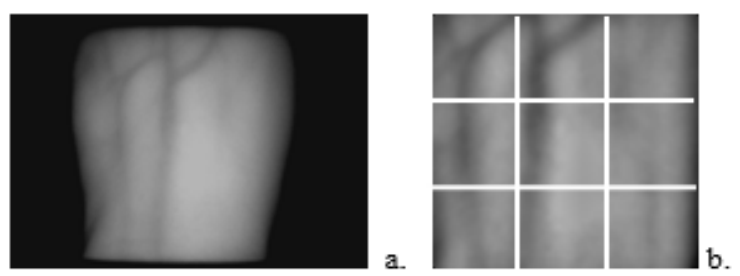

Fig. 6. A. Original image; b. Selected and enhanced ROI.

The ILFOE technique is applied independently on each block to encode the pattern information. Different local features were estimated, from a k-by-k neighborhood, around each reference pixel:

- The range value (maximum value - minimum value) of the neighborhood.

- The median value.

- A pixelwise adaptive Wiener value based on local mean and variance.

- The standard deviation.

- The entropy value.

- The local binary pattern.

- The rotation invariant local binary pattern, etc.

The resulted image is of the same size as the input one for all above mentioned techniques. Different experiments were conducted to select an appropriate combination of individual features for biometric recognition. Best recognition rates were achieved by incorporating local range with median and pixelwise adaptive Wiener values. A symmetric and rectangular 9x9 neighborhood centered at each pixel, yield the most accurate prediction in case of iris based system, while for hand veins system a 5 x 5 neighborhood seems to be the most appropriate.

For gray images, the range, median and Wiener values, extracted from image patches, were further converted into a vector based representation by means of the proposed ordinal encoding procedure. Another modality, adequate to color images, was designed by encoding the same local feature, extracted from R, G, B color channels separately.

On these particular situations, $n=3$ thus $3^{3}=27$ symbols are generated for each sub-image. The final vector consists in $\mathrm{N} * 27$ features, where $\mathrm{N}$ is the number of considered blocks: 8 for iris images and 9 for dorsal hand veins images. It is independently of input image size and was obtained by simply concatenating the local histograms. Since the proposed technique is applied on sub-images (blocks) separately, the possible artifacts (segmentation errors, occlusions, etc.) will influence only the corresponding local vector. The recognition rates are listed in Table I. 
TABLE I. RECOGNITION RATES FOR THE UNIMODAL BIOMETRIC SYSTEMS

\begin{tabular}{|c|c|c|c|c|c|c|c|}
\hline System & $\begin{array}{c}\text { Image } \\
\text { Type }\end{array}$ & Database & Classes & $\begin{array}{c}\text { Local } \\
\text { Features }\end{array}$ & $\begin{array}{c}\text { Feature } \\
\text { Vector } \\
\text { Length }\end{array}$ & $\begin{array}{l}\text { Train } \\
\text { /Test } \\
\text { Ratio } \\
\end{array}$ & $\underset{\%}{\text { Accuracy }}$ \\
\hline \multirow{8}{*}{ IRIS } & \multirow{2}{*}{$\begin{array}{l}\text { Gray } \\
\text { Images }\end{array}$} & CASIA_V1 & 93 & \multirow{2}{*}{$\begin{array}{l}\text { Range } \\
+ \\
\text { Median } \\
+ \\
\text { Wiener }\end{array}$} & $8 * 27$ & $3 / 2$ & 97.84 \\
\hline & & UPOL & 64 & & $8 * 27$ & $2 / 1$ & 96.87 \\
\hline & \multirow{6}{*}{$\begin{array}{l}\text { Color } \\
\text { Images }\end{array}$} & \multirow{6}{*}{ UPOL } & \multirow{6}{*}{64} & \multirow{2}{*}{ Range } & \multirow{2}{*}{$8 * 27$} & $2 / 1$ & 95.31 \\
\hline & & & & & & $1 / 2$ & 90.62 \\
\hline & & & & \multirow{2}{*}{ Median } & \multirow{2}{*}{$8 * 27$} & $2 / 1$ & 100 \\
\hline & & & & & & $1 / 2$ & 99.21 \\
\hline & & & & \multirow{2}{*}{ Wiener } & \multirow{2}{*}{$8 * 27$} & $2 / 1$ & 100 \\
\hline & & & & & & $1 / 2$ & 100 \\
\hline \multirow{2}{*}{$\begin{array}{l}\text { HAND } \\
\text { VEIN }\end{array}$} & \multirow{2}{*}{$\begin{array}{l}\text { Gray } \\
\text { Images }\end{array}$} & \multirow[b]{2}{*}{ NCUT } & \multirow[b]{2}{*}{102} & \multirow{2}{*}{$\begin{array}{l}\text { Range } \\
+ \\
\text { Median } \\
+ \\
\text { Wiener }\end{array}$} & \multirow[b]{2}{*}{$9 * 27$} & $6 / 4$ & 94.85 \\
\hline & & & & & & $7 / 3$ & 96.40 \\
\hline
\end{tabular}

The Support Vector Machine, RBF kernel, has been used for the recognition task since it has been successfully applied in many studies for object classification [23].

\section{B. Bimodal Biometric Systems based on Feature and Score Level Fusions}

A single biometric trait does not satisfy all the requirements (e.g. accuracy, permanence, circumvention, etc.) especially when it comes to large-scale authentication systems [24].

Therefore, a bimodal recognition system based on iris and dorsal hand veins has been also designed, by considering 93 virtual users. Each subject from CASIA_V1 iris database has been combined with a subject belonging to NCUT vein database. The use of virtual subjects is a common and accepted procedure in biometrics.

The fusion has been made at the feature level and matching-score level. The second strategy combines the matching scores of each unimodal system, in order to arrive at a final decision about the users' identity. The scores provided by individual matchers are incorporated by the product rule. The recognition rates are listed in Table II.

The proposed new technique is considered to be suitable for portable applications, especially due to the computationally low costs.

Table III presents comparative summary of several prior approaches presented in the literature for iris respectively dorsal hand vein authentication. For the selected systems, different ordinal based methods have been employed for image analysis.
TABLE II. RECOGNITION RATES FOR THE BIMODAL BIOMETRIC SYSTEM

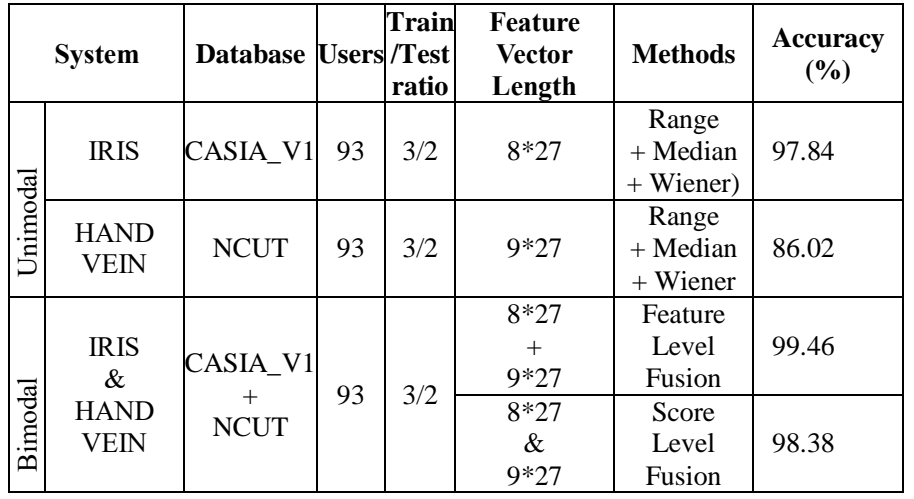

TABLE III. BIOMETRIC SyStEMS BASED ON ORdinAl MEASURES

\begin{tabular}{|c|c|c|c|}
\hline System & Database & Methods & $\begin{array}{c}\text { Accuracy } \\
(\%)\end{array}$ \\
\hline IRIS [25] & CASIA & $\begin{array}{c}\text { LBP } \\
+ \text { combined Learning Vector } \\
\text { Quantization Classifier }\end{array}$ & 99.87 \\
\hline \multirow{2}{*}{ IRIS [11] } & CASIA & \multirow{2}{*}{ Local Intensity Order Pattern (LIOP) } & 96.77 \\
\hline & UPOL & & 100 \\
\hline $\begin{array}{c}\text { HAND VEIN } \\
{[26]} \\
\end{array}$ & NCUT & Partition Local Binary Pattern (PLBP) & 90.88 \\
\hline $\begin{array}{l}\text { HAND VEIN } \\
{[27]}\end{array}$ & NCUT & $\begin{array}{c}\text { Local Binary Pattern } \\
+ \text { geometry features (crossing, end- } \\
\text { points) }\end{array}$ & 96.67 \\
\hline $\begin{array}{l}\text { HAND VEIN } \\
{[8]}\end{array}$ & NCUT & $\begin{array}{c}\text { Riesz Wavelet } \\
+ \text { Local Line Binary Patern } \\
+ \text { Statistical Moments }\end{array}$ & 87.9 \\
\hline
\end{tabular}

\section{CONCLUSIONS}

One fundamental issue in pattern recognition consists in finding a convenient method for image to symbol transformation. The present paper proposes a novel technique resulted by integrating local image features into an ordinal measurement based encoding method.

The obtained results indicate that ILFOF method constitutes a promising solution for image features extraction that could be easily adapted to different matching or recognition tasks. The proposed technique is fast to compute, has a low memory cost and can successfully address real world applications such as biometrics.

Low computational complexity, large tolerance to illumination variations and high degree of accuracy are particular benefits provided by the designed biometric system. Also, fixed length feature vectors are desirable as inputs for the classification module.

To validate the effectiveness and the applicability of the proposed encoding procedure, future work will examine the ILFOF potential on other application area, such as medical imaging. Future research will also explore other methods for image local features extraction.

\section{ACKNOWLEDGMENT}

This work was supported by ANCSI, through the Core Program, project no. PN 16300102; 19N/2016. 


\section{REFERENCES}

[1] He, Dong-Chen, and Li Wang. "Texture unit, texture spectrum, and texture analysis." IEEE transactions on Geoscience and Remote Sensing 28.4 (1990): 509-512

[2] T. Ojala, M. Pietikäinen, and D. Harwood, "A Comparative Study of Texture Measures with Classification Based on Feature Distributions", Pattern Recognition, vol. 19(3), 1996, pp. 51-59.

[3] T. Ojala, M. Pietikäinen, and T. Mäenpää, "Multiresolution gray-scale and rotation invariant texture classification with local binary patterns", IEEE Transactions on Pattern Analysis and Machine Intelligence, vol. 24(7), 2002, pp. 971-987.

[4] S. Emerich, R. Măluţan, E. Lupu and L. Lefkovits "Patch based descriptors for iris recognition", IEEE Conference on Intelligent Computer Communication and Processing (ICCP), 2016, pp. 187-191

[5] Petpon, Amnart, and Sanun Srisuk. "Face recognition with local line binary pattern." Image and Graphics, 2009. ICIG'09. Fifth International Conference on. IEEE, 2009.

[6] Rosdi, Bakhtiar Affendi, Chai Wuh Shing, and Shahrel Azmin Suandi. "Finger vein recognition using local line binary pattern." Sensors 11.12 (2011): 11357-11371.

[7] J. Yusmah, C. Fatichah, and N. Suciati. "Local Line Binary Pattern For Feature Extraction on Palm Vein Recognition." Jurnal Ilmu Komputer dan Informasi 8.2 (2015): 111-118.

[8] Raul Malutan, Simina Emerich, Septimiu Crisan, Olimpiu Pop, László Lefkovits, "Dorsal Hand Vein Recognition Based on Riesz Wavelet Transform and Local Line Binary Pattern", International Conference on Frontiers of Signal Processing (ICFSP 2017) France, 2017.

[9] Z. Wang, B. Fan, and F. Wu, "Local intensity order pattern for feature description," IEEE International Conference On Computer Vision (ICCV), 2011, pp. 603-610,

[10] Simina Emerich, Raul Malutan, Septimiu Crisan, László Lefkovits, 'Iris Indexing based on Local Intensity Order Pattern', The 9th International Conference on Machine Vision (ICMV 2016), November 18-20, 2016, Nice, France

[11] Raul Malutan, Simina Emerich, Olimpiu Pop, László Lefkovits, "Half Iris Biometric System based on HOG and LIOP", 2016 2nd International Conference on Frontiers of Signal Processing (ICFSP 2016), October, 15-17, 2016, Warsaw, Poland, IEEE, ISBN 978-1-5090-3814-5

[12] Xu, X., Tian, L., Feng, J., Zhou, J.: "OSRI: a rotationally invariant binary descriptor." IEEE Trans. Image Process. 23(7), 2983-2995 (2014)

[13] Fan, B., Wu, F., Hu, Z.: "Rotationally invariant descriptors using intensity order pooling." IEEE Trans. Pattern Anal. Mach. Intell. 34(10), 2031-2045 (2012)
[14] Fan, Bin, Z. Wang, and Fuchao Wu. "Local Image Descriptor: Modern Approaches. " Springer, 2016

[15] Chatfield, K., Lempitsky, V. S., Vedaldi, A., \& Zisserman, A. "The devil is in the details: an evaluation of recent feature encoding methods." In BMVC, Vol. 2, No. 4, p. 8, 2011

[16] Huang, Yongzhen, and Tieniu Tan. "Feature Coding for Image Representation and Recognition“, Springer Berlin Heidelberg, 2014.

[17] Brahnam, S., Jain, L. C., Nanni, L., \& Lumini, A. "Local binary patterns: new variants and applications." Springer Berlin Heidelberg, 2014

[18] King, Reginald Alfred. "Waveform coding method", U.S. Patent No 6.748.354, 2004.

[19] M Dobeš, J. Martinek, D. Skoupil, Z. Dobešová, and J. Pospíšil, "Human eye localization using the modified Hough Transform," in Optik, vol. 117(10), Eds. Elsevier, 2006, pp. 468-473.

[20] CASIA-IrisV1, http://biometrics.idealtest.org/.

[21] R. Arun, and S. Shah, "Segmenting non-ideal irises using geodesic active contours," IEEE Biometrics Symposium: Special Session on Research at the Biometric Consortium Conference, Sep. 2006.pp. 1-6, doi:10.1109/BCC.2006.4341625.

[22] Wang, Yiding, Kefeng Li, and Jiali Cui. "Hand-dorsa vein recognition based on partition local binary pattern." Signal Processing (ICSP), 2010 IEEE 10th International Conference on. IEEE, 2010

[23] Apatean, A., Rusu, C., Rogozan, A., \& Bensrhair, A. "Visible-infrared fusion in the frame of an obstacle recognition system", IEEE International Conference on Automation Quality and Testing Robotics (AQTR), Vol. 1, 2010, pp. 1-6

[24] Kekre, H. B., Tanuja Sarode, and Rekha Vig. "Multi-resolution analysis of multi-spectral palmprints using hybrid wavelets for identification." International Journal of Advanced Computer Science and Applications (IJACSA) 4.3, 2013, pp. 192-198

[25] Shams, M. Y., Rashad, M. Z., Nomir, O., \& El-Awady, R. M. "Iris recognition based on LBP and combined LVQ classifier", International Journal of Computer Science \& Information Technology (IJCSIT) Vol 3, No 5, 2011, pp 67-78

[26] Wang Y., Li K., Cui J., Shark LK., Varley M. "Study of Hand-Dorsa Vein Recognition". In: Huang DS., Zhao Z., Bevilacqua V., Figueroa J.C. (eds) Advanced Intelligent Computing Theories and Applications. ICIC 2010. Lecture Notes in Computer Science, vol 6215, pp. 490-498 Springer, Berlin, Heidelberg

[27] Zhu X., Huang D. "Hand Dorsal Vein Recognition Based on Hierarchically Structured Texture and Geometry Features" In: Zheng WS., Sun Z., Wang Y., Chen X., Yuen P.C., Lai J. (eds) Biometric Recognition. CCBR 2012. Lecture Notes in Computer Science, vol 7701, pp. 157-164, Springer, Berlin, Heidelberg 